\title{
From High Accuracy to High Efficiency in Simulations of Processing of Dual-Phase Steels
}

\author{
L. RAUCH, R. KUZIAK, and M. PIETRZYK
}

Searching for a compromise between computing costs and predictive capabilities of metal processing models is the objective of this work. The justification of using multiscale and simplified models in simulations of manufacturing of DP steel products is discussed. Multiscale techniques are described and their applications to modeling annealing and stamping are shown. This approach is costly and should be used in specific applications only. Models based on the JMAK equation are an alternative. Physical simulations of the continuous annealing were conducted for validation of the models. An analysis of the computing time and predictive capabilities of the models allowed to conclude that the modified JMAK equation gives good results as far as prediction of volume fractions after annealing is needed. Contrary, a multiscale model is needed to analyze the distributions of strains in the ferritic-martensitic microstructure. The idea of simplification of multiscale models is presented, as well.

DOI: $10.1007 / \mathrm{s} 11663-013-9926-5$

(C) The Author(s) 2013. This article is published with open access at Springerlink.com

\section{INTRODUCTION}

A numerical simulation of phase transformations to obtain two-phase microstructures and analysis of deformation of these microstructures is the objective of the work. Historically, models based on the JMAK equation were commonly used in the 20th century for simulations of processing of steels. Since early 1970s, finite-element (FE) method has become the most popular simulation technique as far as flow and temperatures during processing are considered. In the 1990s, the FE codes were connected with the microstructure evolution models and thermal-mechanical-microstructural simulations became possible. New challenges in modeling metal processing occurred in the 21st century. The possibility of the prediction of material behavior accounting explicitly for the granular structure of polycrystals was the first challenge, which led to development of multiscale models. In these models, usually FE codes are connected with such discrete methods as cellular automata (CA), molecular dynamics (MD), or Monte Carlo (MC). The problem of computing time is the second challenge, which is particularly important when optimization of the process is needed. Models based on closed-form equations are still used to enable efficient application of advanced optimization techniques, which require a large number of calculations of the objective function. Thus, searching for a compromise between computing time and predictive capabilities of phase transformation and stamping models is the objective of this work. Justification of using

L. RAUCH, Assistant Professor, and M. PIETRZYK, Professor, are with the AGH University of Science and Technology, 30-059 Krakow, Poland. Contact e-mail: lrauch@agh.edu.pl R. KUZIAK, Manager, is with the Institute for Ferrous Metallurgy, 44-100 Gliwice, Poland.

Manuscript submitted February 11, 2013.

Article published online September 20, 2013. multiscale and simplified models in processing DP steels is discussed.

\section{CLASSIFICATION OF MODELS}

The classification of phase transformation models with respect to predictive capabilities and computing time is shown in Figure 1. Models in the left bottom corner are used mainly for basic simulations and optimization of processes or whole manufacturing cycles. Historically, the JMAK equation ${ }^{[1-3]}$ and its modifications ${ }^{[4]}$ were commonly used for fast simulations of phase transformations.

The models in the center of Figure 1 are based on the FE or phase-field method, and they are used in the technology design and optimization of processes. The latter approach has emerged as one of the most powerful methods for modeling various microstructure evolution processes, including the austenite decomposition. A description of this method was presented in References 5 and 6. Briefly, the phase-field model treats a polycrystalline system, containing both bulk and boundary regions, in an integral manner. A set of continuous phase-field variables, each representing an individual grain, is defined to have a constant value inside the grains and will change continuously over a diffuse boundary. The driving force is calculated from the local carbon composition within the interface, controlled by the diffusion in the austenite. A specific feature of the phase-field approach is that the boundary between two distinct regions is diffused. Therefore, the computational complexity of the phase-field model is comparable to the deterministic model based on the direct solution of the diffusion equation.

In the 1990s, connection of the JMAK equation to FE codes created a possibility to predict the distribution of microstructural parameters in the volume of products. ${ }^{[7]}$ 
The need for simulation of microstructural phenomena accounting explicitly for the structure of polycrystals led to the development of multiscale models located in the top right corner in Figure 1.

The microstructure can also be explicitly accounted for in modeling phase transformation when the FE solution of the diffusion equation with moving boundary is applied. ${ }^{[8]}$ This solution supplies information about carbon distribution in the austenite. In consequence, predicting the hardness distribution in martensite islands becomes possible.

In multiscale modeling, usually FE codes are connected with such discrete methods as CA, MD, or MC. A review of multiscale modeling methods can be found in Reference 9. The problem of computing time is the challenge, which is particularly important when optimization of the process is performed. Multiscale methods give better insight in the phenomena occurring at lower dimensional scales, but they require long computing times.

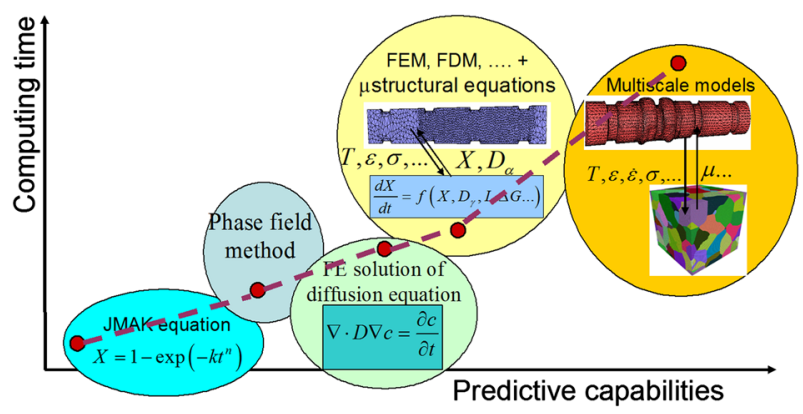

Fig. 1-Classification of phase-transformation models with respect to their predictive capabilities and computing time.

Table I. Additional Relations in the Phase Transformation Model Based on the JMAK Eq. [1]

$c_{\gamma}=\frac{\left(c_{0}-X_{f} c_{\alpha}\right)}{1-X_{f}} X_{f 0}=\frac{c_{\gamma \alpha}-c_{0}}{c_{\gamma \alpha}-c_{\alpha}}$

$c_{\gamma \alpha}=4.77968-0.005782 T$

$c_{\gamma \beta}=-0.85633+0.002167 T$

$B_{s}=a_{20}-425[\mathrm{C}]-42.5[\mathrm{Mn}]-31.5[\mathrm{Ni}]-70[\mathrm{Cr}]$

$M_{s}=a_{26}-a_{27} c_{\gamma}$

$X_{m}=1-\exp \left[-0.011\left(M_{s}-T\right)\right]$

$F_{m}=\left(1-F_{f}-F_{p}-F_{b}\right) X_{m}$
This article presents the results of research on the improvement of the accuracy of the JMAK equation, as well as on a decrease of the computing time for multiscale modeling techniques. The former was achieved by introduction of relations of coefficients on the temperature. The latter was achieved by introduction of the statistically similar representative volume element (SSRVE).

\section{PHASE TRANSFORMATION MODEL}

The model is based on the JMAK equation:

$$
X=1-\exp \left(-k t^{n}\right)
$$

where $t$ is the time and $X$ is the transformed volume fraction.

Equation [1] is combined with the Scheil additivity rule, which accounts for the temperature variations. Theoretical considerations show that a constant value of $n$ in Eq. [1] can be used. This coefficient is introduced further in the vector a of the model coefficients containing $a_{4}, a_{16}$, and $a_{24}$ for ferritic, pearlitic, and bainitic transformations, respectively. On the contrary, the value of the coefficient $k$ must vary with temperature. The formalism of the function $k=\mathrm{f}(T)$ must be carefully chosen to describe properly the temperature dependence of transformation kinetics. This problem was thoroughly discussed in Reference 10. A sensitivity analysis was performed and the best functions $k=\mathrm{f}(T)$ were selected. All equations of the final version of the model are given in Tables I and II.

The simulation of ferritic transformation begins with Eq. [1] when the temperature drops below $\mathrm{Ae}_{3}$. The transformed volume fraction $X_{f}$ is calculated with respect to the maximum volume fraction of ferrite $X_{f 0}$ at the current temperature calculated with ThermoCalc (Thermo-Calc Software, Stockholm, Sweden). Thus, this volume fraction with respect to the whole volume of the body is $F_{f}=X_{f} X_{f 0}$. During simulation in the varying temperature, the current value of $X_{f}$ calculated from Eq. [1] has to be corrected to account for the change of the maximum volume fraction of ferrite $X_{f 0}$, which according to equation in Table $\mathrm{I}$ is a function of temperature.

Because continuous annealing in the intercritical region was selected as a case study in the current work,

Table II. Additional Equations for Ferritic, Pearlitic, and Bainitic Transformations

\begin{tabular}{lll}
\hline Parameter & Ferrite & Pearlite \\
\hline$k=\mathrm{f}(T)$ & $k=\frac{a_{5}}{D_{\gamma}} \exp \left[-\left(\frac{T-A e_{3}-\frac{400}{D_{\gamma}}+a_{6}}{a_{8}}\right)^{a_{7}}\right]$ & $k=a_{12}$ \\
$\begin{array}{l}\text { Incubation } \\
\text { time }\end{array}$ & - & $\tau_{P}=\frac{a_{9}}{\left(A e_{1}-T\right)^{a_{11}}} \exp \left[\frac{a_{10}}{R(T+273)}\right]$
\end{tabular}

Notation: $c_{\gamma}$, is the average carbon content in the austenite; $c_{\alpha}$ is the carbon content in the ferrite; $c_{0}$ is the carbon content in the steel; $c_{\gamma \alpha}$ is the carbon concentration in the austenite at the $\gamma-\alpha$ boundary; $\mathrm{c}_{\gamma \beta}$ is the carbon concentration in austenite at the $\gamma$-cementite boundary; $X_{f 0}$ is the maximum volume fraction of ferrite according to equilibrium calculation in the current temperature; $B_{\mathrm{s}}$ is the bainite start temperature; $M_{\mathrm{s}}$ is the martensite start temperature; $X_{f}, X_{\mathrm{m}}$ are the volume fractions of ferrite and martensite with respect to the maximum volume fraction of this phases; $F_{f}, F_{b}, F_{\mathrm{m}}$ are the volume fractions of ferrite, bainite, and martensite with respect to the whole volume of the material; and $D_{\gamma}$ is the austenite grain size at the beginning of transformation represented by mean linear intercept. 
transformation of the ferritic-pearlitic microstructure into the austenite has to be also considered. The kinetics of the austenitic transformation during heating is described by Eq. [1] with the coefficient $n=a_{30}$ and the coefficient $k$ defined as:

$$
k=a_{28} \exp \left[\frac{a_{29}}{R(T+273)}\right]
$$

The incubation time for the ferrite-austenite transformation is calculated from the equation:

$$
\tau_{a}=\frac{a_{1}}{\left(T-\mathrm{A} e_{1}\right)^{a_{3}}} \exp \left[\frac{a_{2}}{R(T+273)}\right]
$$

The full model contained 30 coefficients grouped in the vector a. After the sensitivity analysis, ${ }^{[10]}$ the model was simplified and 26 coefficients remained. These coefficients are determined using an inverse analysis of dilatometric tests.

\section{EXPERIMENT AND IDENTIFICATION}

Dual-phase (DP) steel containing 0.1 pct C, 1.51 pct $\mathrm{Mn}, 0.23$ pet $\mathrm{Si}, 0.41 \mathrm{Cr}, 0.05$ pet $\mathrm{Mo}, 0.045$ pet V, 0.006 pet Ti, 0.007 pet $\mathrm{P}, 0.007$ pet $\mathrm{S}, 0.037$ pet Al, and 0.009 pet $\mathrm{N}$ was investigated. Experiments involving dilatometric tests during heating and cooling were performed to supply data for identification of the models. Samples measuring $1.4 \times 2.2 \times 7 \mathrm{~mm}$ were cut from a cold-rolled strip. In the first set of tests, samples were heated to the selected temperature at a rate of $3 \mathrm{~K}\left(3{ }^{\circ} \mathrm{C}\right) / \mathrm{s}$ and fast cooled at a rate of approximately $3 \mathrm{~K}\left(180{ }^{\circ} \mathrm{C}\right) / \mathrm{s}$ with helium. The microstructure was analyzed with Neophot 2 light microscope and Inspect field emission gun electron microscope, and volume fractions of phases were evaluated using the Metilo program for automatic image analysis. In the second set of experiments, the samples were austenitized at $1163 \mathrm{~K}\left(890{ }^{\circ} \mathrm{C}\right)$ and cooled with various cooling rates. An inverse analysis preceded by the sensitivity analysis was applied for the identification of the coefficients in the model. Details of the sensitivity analysis for the phase transformation model are given in Reference 10. The inverse algorithm proposed in Reference 11 and applied to phase transformation model in Reference 12 was used in the current work. Briefly, a mathematical model of an arbitrary process or physical phenomenon can be described by a set of equations:

$$
\mathbf{d}=F(\mathbf{a}, \mathbf{p}), \quad F: R^{k+l} \rightarrow R^{r}
$$

where $\mathbf{d}=\left\{d_{1}, \ldots, d_{r}\right\}$ is the vector of output variables of the model (start and end of transformations temperatures, volume fractions), $\mathbf{a}=\left\{a_{1}, \ldots, a_{k}\right\}$ is the vector of coefficients of the model, and $\mathbf{p}=\left\{p_{1}, \ldots, p_{l}\right\}$ is the vector of the known process parameters (cooling rates). When vectors $\mathbf{p}$ and $\mathbf{a}$ are known, the solution of the Eq. [4] is called a direct solution. The inverse solution of Eq. [1] is defined as determination of the
Table III. Coefficients in the Phase Transformation Model Calculated Using Inverse Analysis for the Investigated DP Steel

\begin{tabular}{lllllll}
\hline$a_{1}$ & $a_{2}$ & $a_{3}$ & $a_{4}$ & $a_{5}$ & $a_{6}$ & $a_{7}$ \\
1039 & 4.861 & 2.866 & 2.119 & 0.699 & 148.2 & 55.35 \\
$a_{8}$ & $a_{9}$ & $a_{10}$ & $a_{11}$ & $a_{12}$ & $a_{16}$ & $a_{17}$ \\
2.141 & 75.01 & 2.124 & 0.579 & 1.0 & 1.285 & 1600 \\
$a_{18}$ & $a_{19}$ & $a_{20}$ & $a_{21}$ & $a_{22}$ & $a_{23}$ & $a_{24}$ \\
64.64 & 3.495 & 715.8 & 2.485 & 0.374 & 2.5 & 4.038 \\
$a_{26}$ & $a_{27}$ & $a_{28}$ & $a_{29}$ & $a_{30}$ & & \\
435.0 & 2.326 & 9636 & 79.4 & 0.229 & & \\
\hline
\end{tabular}

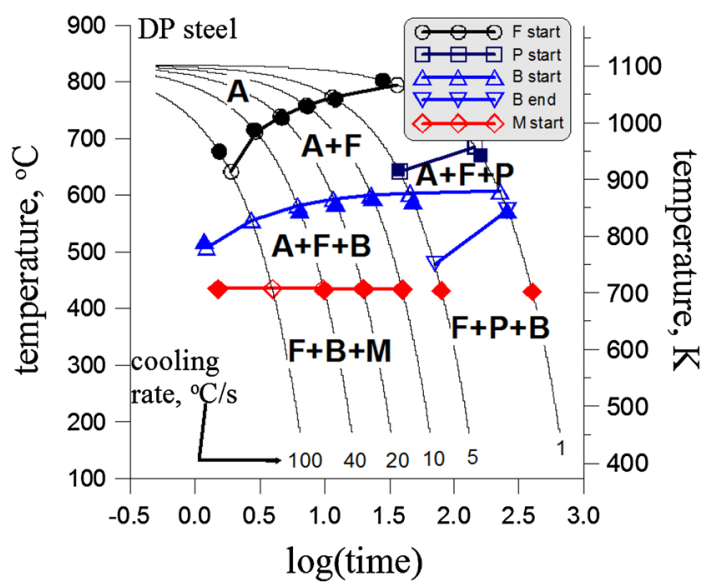

(a)

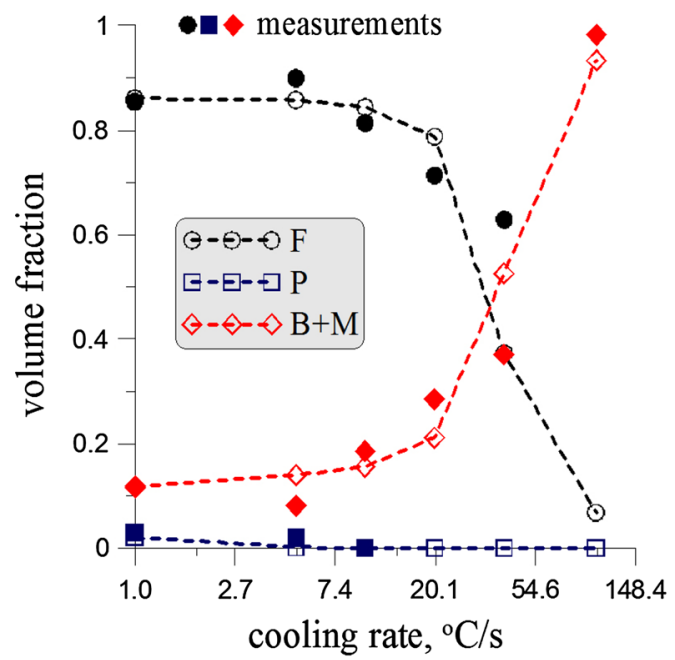

(b)

Fig. 2-Comparison of the measured (filled symbols) and calculated (open symbols with dotted lines) CCT diagrams $(a)$ and volume fractions of phases $(b)$.

components of the vector a for known vectors $\mathbf{d}$ (from dilatometric tests) and $\mathbf{p}$ :

$$
\mathbf{a}=F^{-1}(\mathbf{d}, \mathbf{p}), \quad F^{-1}: R^{r+l} \rightarrow R^{k}
$$

When the problem is linear, the inverse function can be usually found and the problem can be solved 
analytically. In the investigated problem, this relation is nonlinear and the problem is transformed into the optimization task. Thus, the objective of the inverse analysis is to determine the optimum components of vector a by searching for the minimum, with respect to the components of this vector, of the objective function

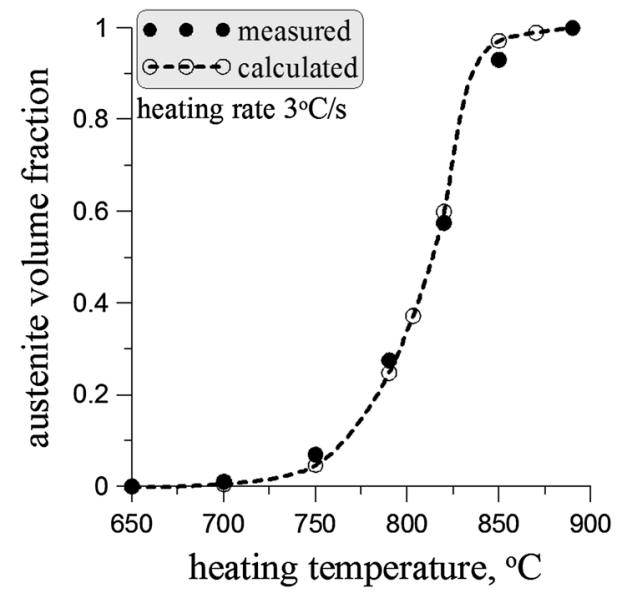

Fig. 3-Measured and calculated kinetics of transformation during heating. defined as a square root error between measured and calculated components of the vector $\mathbf{d}$ :

$$
\Phi(\mathbf{a}, \mathbf{p})=\sum_{i=1}^{n} \beta_{i}\left[\mathbf{d}_{i}^{c}\left(\mathbf{a}, \mathbf{p}_{i}\right)-\mathbf{d}_{i}^{m}\right]^{2}
$$

where $\mathbf{d}_{i}^{m}$ is a vector containing the start and end of transformations temperatures and volume fractions of phases measured in dilatometric tests, $\mathbf{d}_{i}^{c}$ is a vector containing these parameters calculated by the model, $\beta_{i}$ is the weights of the points, $(i=1, \ldots, n)$, and $n$ is the number of measurements. The values of the coefficients given in Table III were obtained from the inverse analysis of dilatometric tests.

The model with the coefficients in Table III was validated. All performed dilatometric tests were simulated with the developed model. Figure 2 shows a comparison of the measured (filled symbols) and predicted (open symbols) start and end temperatures for the phase transformations and volume fractions of phases. The shape of the symbol refers to the temperature or volume fraction in the legend. An analysis of the results shows that the model predicts well the start and end temperatures for the transformations. The accuracy is worse for the bainite end temperature only, possibly because of difficulties with a very accurate evaluation of
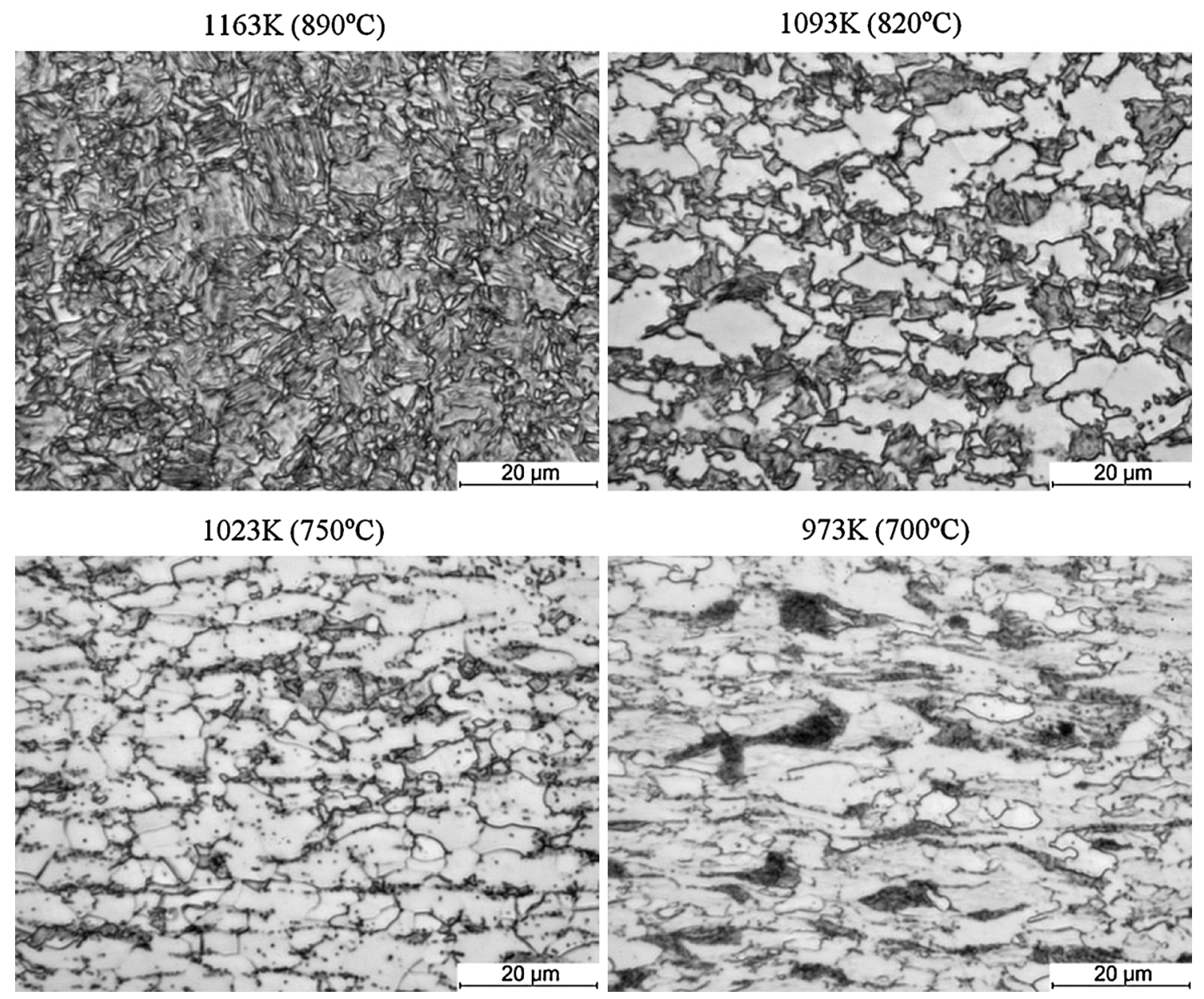

Fig. 4-Microstructures after heating to various temperatures. 
this temperature from the dillatometric data. In the experiment, the martensite start temperature was interpreted as the end of bainitic transformation, while the model predicts the end of this transformation at higher temperature.

The volume fractions of phases are properly predicted for slower cooling rates (below $25 \mathrm{~K}\left[25^{\circ} \mathrm{C}\right] / \mathrm{s}$ ). There were some difficulties in distinguishing between bainite and martensite for high cooling rates, but good agreement between measurement and calculations was observed for the sum of the volume fractions of hard components. This is because the transition between bainite and martensite is not predicted accurately with the developed model. One may assume that this is an inherited feature of the conventional models based on the JMAK model. There is also a significant influence of the inaccuracies of the ferrite and pearlite transformation predictions on the further start and kinetics of bainite and martensite transformation because in the model the mean carbon content at the end of diffusional transformations is calculated to predict $B_{\mathrm{s}}$ and $M_{\mathrm{s}}$ temperatures. One must also consider the fact that in a real process, the carbon may not be evenly distributed in the austenite as is assumed in the current model.

The phase transformation model for heating was validated next. Figure 3 shows comparison of measured and calculated volume fraction of the austenite after heating to various temperatures. The measurement of the austenite volume fraction was performed using a dilation curve obtained in the dilatometric test. Selected examples of microstructure after heating for these tests are shown in Figure 4.

\section{CONTINUOUS ANNEALING}

Simulations of the continuous annealing process were conducted for testing the models. Four arbitrary annealing thermal cycles (dotted lines and open symbols in Figure 5(a)) were simulated and the calculated volume fractions are shown in Figure 5(b). It was concluded that although these cycles gave good results for other chemistries of DP steels, see for example Reference 10, the ferritic transformation was too fast for the chemistry investigated in this study. After numerical tests for various cycles, it was possible to select the one that gives less than 80 pct of the ferrite in the microstructure, the martensite as the main hard constituent and small amount of bainite (cycle A5 in Figure 5).

This thermal cycle was reproduced in the DIL 805 dilatometer. The microstructure of the sample is shown in Figure 6. It is composed of ferrite and hard constituents. The volume fraction of the hard constituent is approximately 0.32 , of which around 0.07 can be identified as bainite and the rest is martensite or autotempered martensite. The examples of martensite and bainite are shown in Figure 7 . The result is in good qualitative agreement with the developed schedule using the phase transformation model. The volume fraction of martensite was predicted quantitatively well.

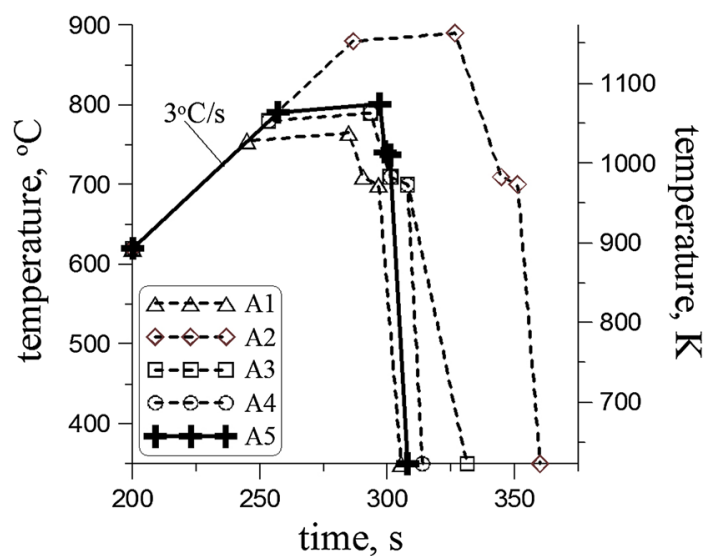

(a)

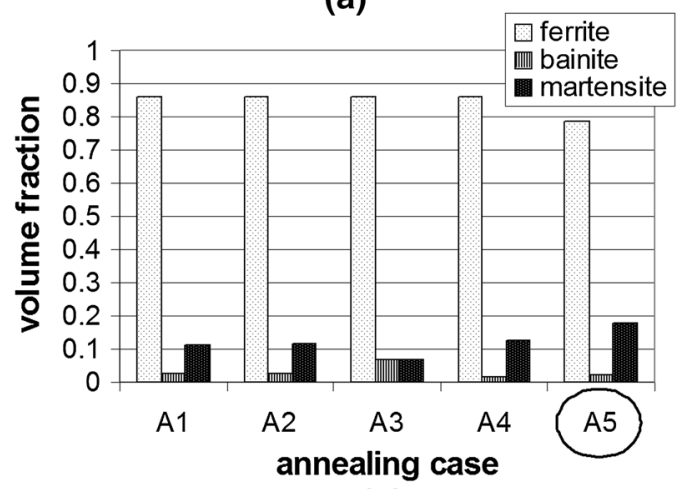

(b)

Fig. 5-Investigated thermal cycles for the continuous annealing $(a)$ and volume fractions of ferrite, bainite, and martensite for these cycles $(b)$.

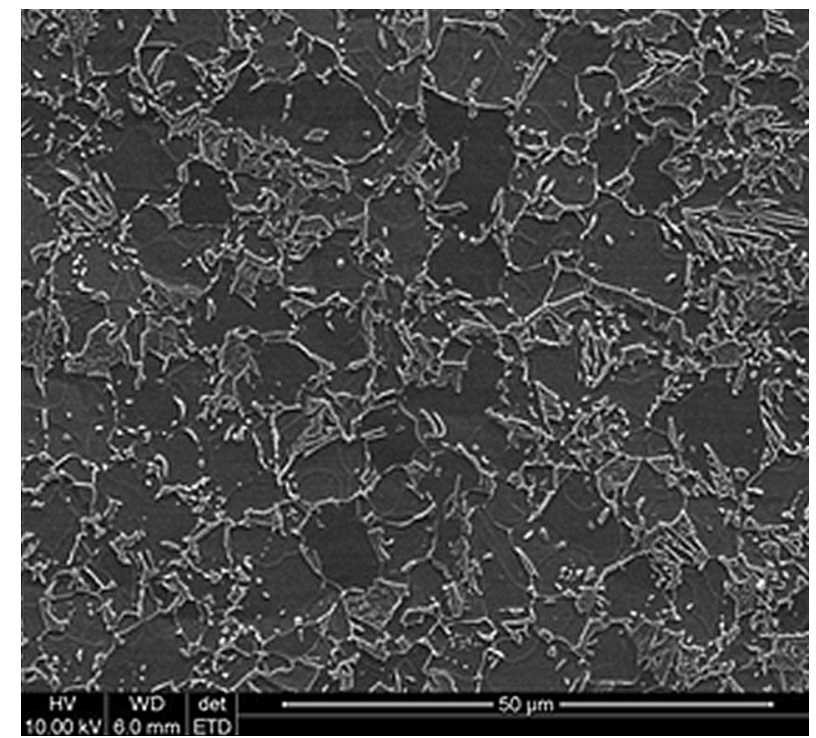

Fig. 6-Microstructures after annealing cycle A5 containing 32 pct of hard constituents, about 25 pct of martensite, and 7 pct of bainite.

Numerical tests and physical simulations confirmed good predictive capability of the model based on the modified JMAK equation as far as volume fractions of 

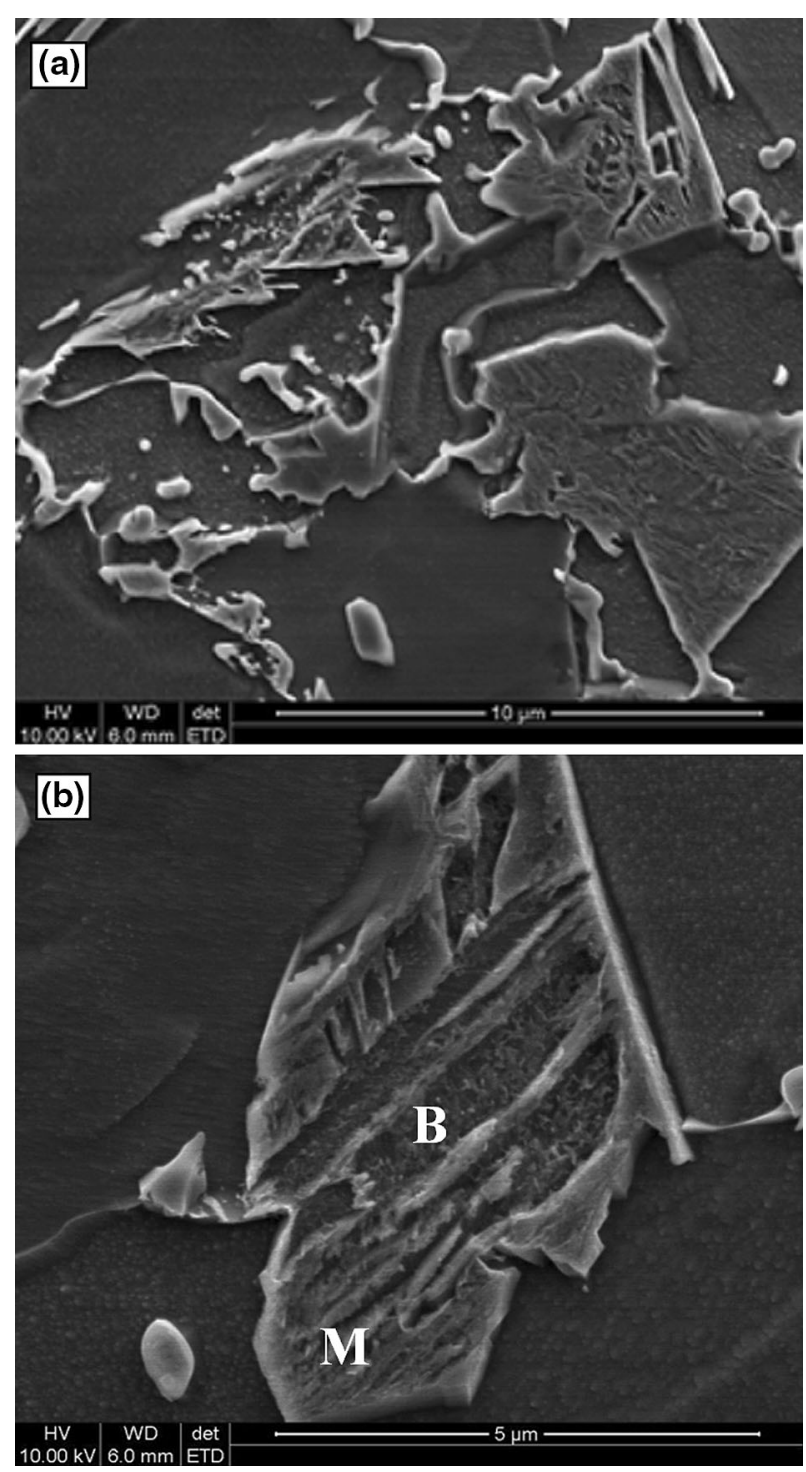

Fig. 7-Example of martensite island (a) and martensite + bainite component $(b)$ observed in the microstructures after annealing cycle A5. phases are considered. The capabilities and computing times for multiscale models are discussed below.

\section{SIMPLIFIED MULTISCALE MODEL}

Multiscale techniques are now commonly used for modeling of phase transformations; see Authors Cellular Automata model for DP steels. ${ }^{[13]}$ However, even for the small size of CA spaces, this approach requires long computing times. The possibility of simplification of the multiscale model is discussed below.

\section{A. Idea of the RVE and SSRVE}

The smallest possible subdomain, which still can represent the macroscopic behavior of the material, is representative volume element (RVE). Although by definition it is the smallest possible piece of material, it still can be too complex for efficient calculations. Therefore, the current research is focused on developing methods allowing a strong reduction of computing time. This led to the construction of SSRVE, which are characterized by a lower complexity than the smallest possible substructure. ${ }^{[14]} \mathrm{A}$ schematic illustration of the idea of the SSRVE is presented in Figure 8.

The objective is to replace an RVE with an arbitrary complex inclusion morphology by an optimal unit cell composed of one (two or maximum three) martensite islands. This simplified SSRVE is repeated periodically in the microstructure, and in consequence, it behaves under loading conditions in the same way as the original complex RVE. This approach allows to decrease the computing time and to make the micro-macro modeling approach more efficient. There is no precise definition of the SSRVE in literature; however, it has to be understood as a highly simplified RVE with a small number of artificially created inclusions and similar behavior under loading. Such a structure is statistically characterized by the same features. The creation of SSRVE on the basis of $\mathrm{RVE}$ is based on optimization procedures joined
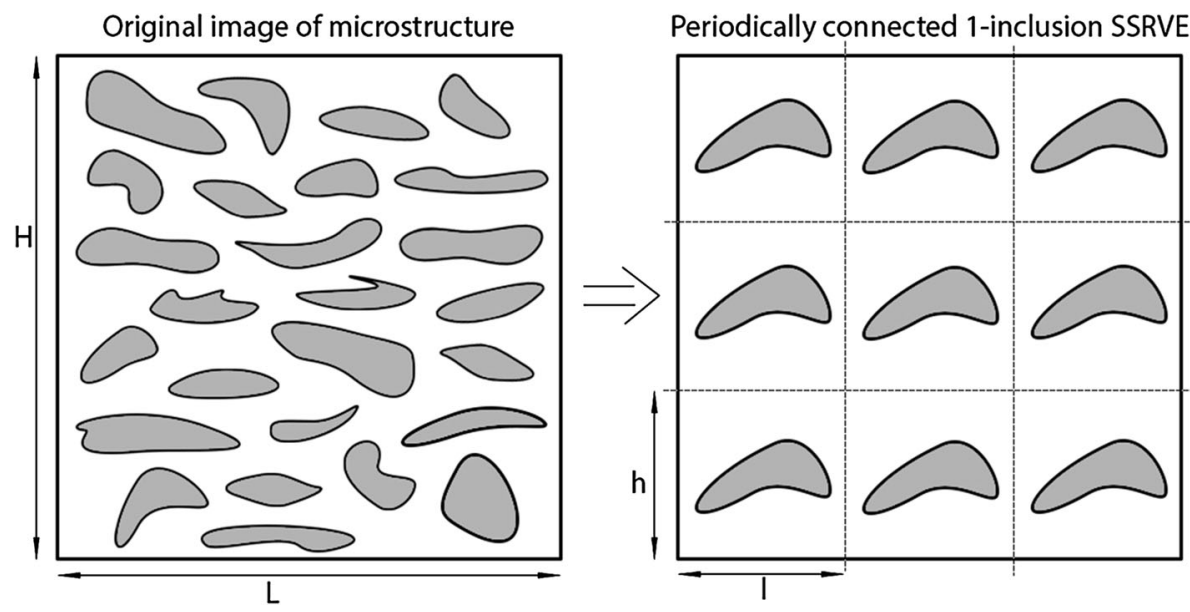

Fig. 8-Schematic illustration of the idea of the SSRVE. 
together with image-processing methods and an analysis of shape coefficients of martensite islands.

\section{B. Creation of the SSRVE}

The procedure of the SSRVE creation is an optimization technique that starts with image recognition algorithms. It usually consists of the following two major steps, applied on original microstructure:

- Filtering: The first step of an analysis aims at phase and edge detection to obtain images with clearly separated phases. It is required to use one of the binarization algorithms as a preconditioner to get a resultant image containing only black and white areas. This procedure is applied not only in the case of multiphase metals but also in the case of alloys. ${ }^{[15]}$ The presented approach of the SSRVE creation assumes that an analyzed phase is always black and the background is white.

- Segmentation: Before shape factors estimation, it is necessary to separate the inclusions from each other because some inclusions are usually joined together by thin artefacts related to ferrite grain boundaries, scratches, etc. ${ }^{[16]}$ The separation is performed by using segmentation methods, which can be divided into four following groups ${ }^{[17]}$ : convolution, nature inspired, diffusion based, and clustering based. Usually in the case of dual-phase micrographics, connected areas are processed subsequently by morphology-based image filtering methods, e.g., erosion or dilation, and by grain boundary detection algorithms to separate particular grains within each phase. The ferrite grain boundaries are not important in this application, and the whole analysis is focused in the inclusion phase. The shape coefficients, which characterize the inclusion, are calculated and used to design the SSRVE. The results of the segmentation of the microstructure investigated in the current work are presented in Figure 9.

After image processing, each segmented element of the original image is identified as a separated material grain. Shape coefficients, calculated for each grain, are collected within a set of 13 coefficients including, e.g., ellipsoid fit, roundness, or curvature. The numerical procedures for the most of the coefficients are based on a body and contour analysis of grouped pixels. The obtained results are gathered into histograms, which are the input for sensitivity analysis. This allows to determine so-called reference values, which are the basis of the objective function in the main SSRVE optimization procedure. Details of the procedure proposed by the authors can be found in Reference 16. Examples of
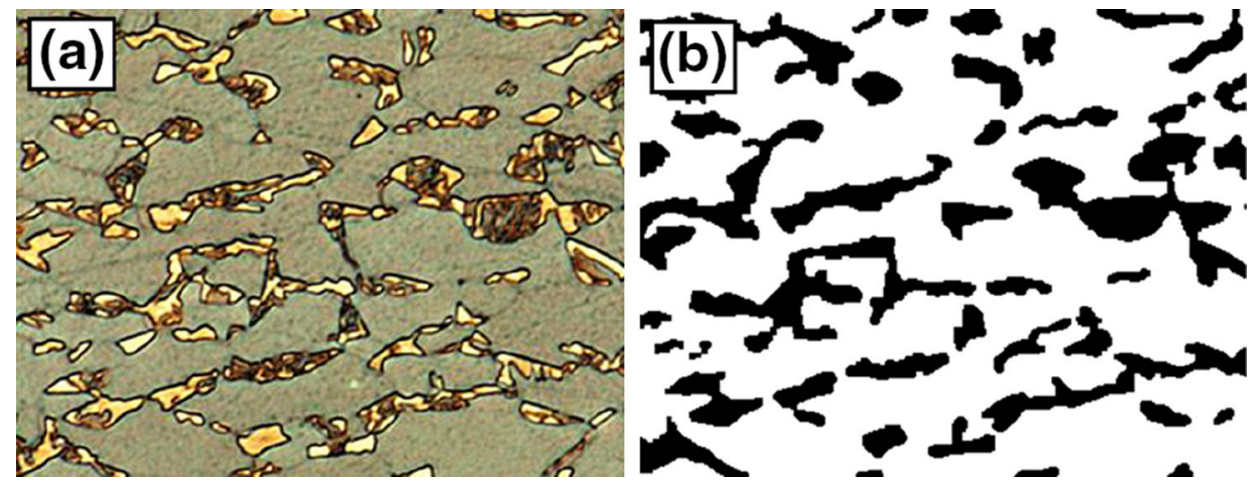

Fig. 9- Original (a) and segmented/binarized (b) images of microstructure of HTC600 steel.
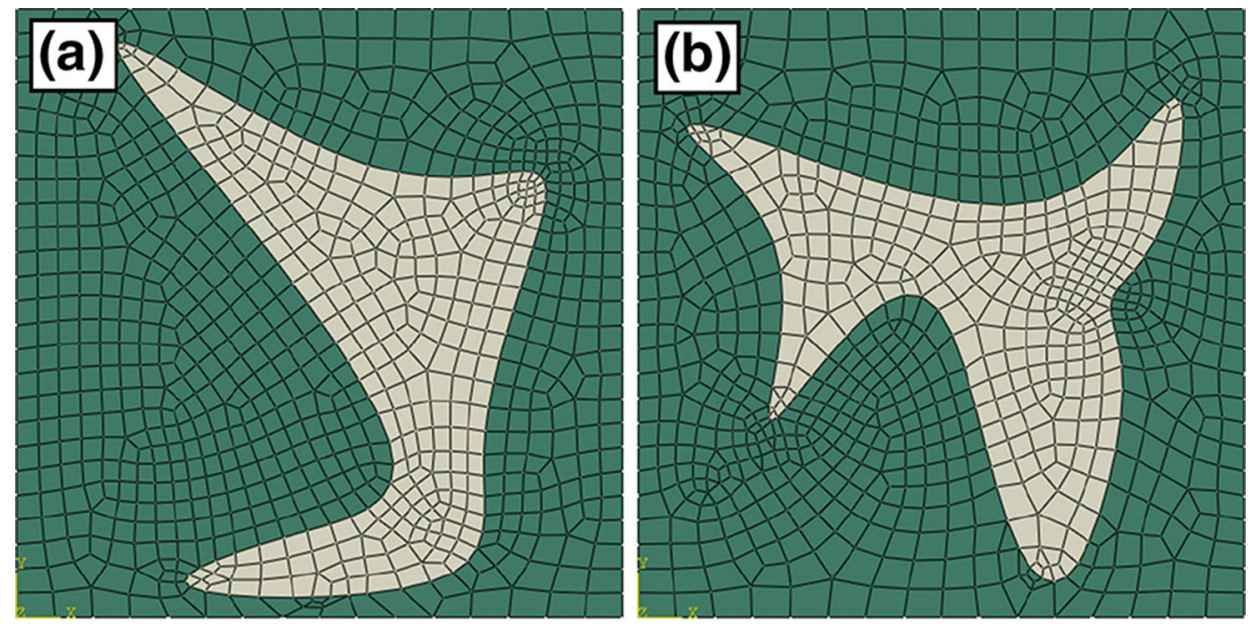

Fig. 10-Examples of SSRVEs, composed of 10 and 13 reference points - (a) and (b), respectively. 
two SSRVEs obtained for HTC600 DP steel are shown in Figure 10.

\section{Simulation of Stamping}

Multiscale simulations with the proposed SSRVE approach were applied to analyze real industrial problems. One of these applications was stamping of crash box, ${ }^{[18]}$ which is widely used in automotive industry because of its high influence on passengers safety during road accidents. It is produced of new generation advanced high strength steels (AHSS), which require a precisely controlled manufacturing process. Nevertheless, this process, which is composed of several steps of material forming, is very difficult for reliable numerical modeling and may be very computationally demanding. This mainly depends on the number of calculations in microscale, which are related to areas in material, where the strain of highest value is obtained in macroscale. Such places usually require a detailed analysis.

In all points of interest, a unit volume element $\left(V=1 \mathrm{~mm}^{3}\right)$ is defined, where various SSRVEs are attached. The displacements from these areas are selected and passed to a two-dimensional (2D) representation of microstructure. In modeling of deformation of the 2D SSRVE, the third component of strain is introduced. This component is determined from the

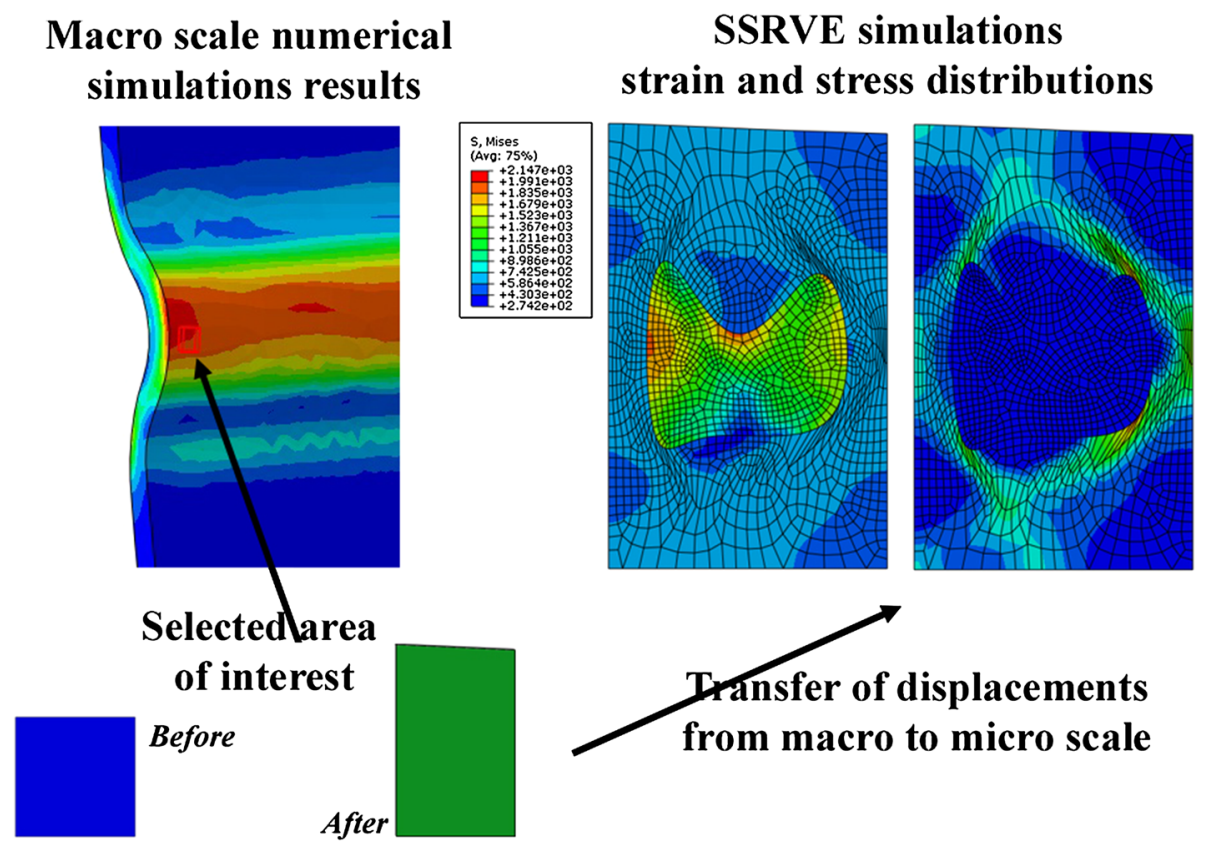

Fig. 11-Idea of SSRVE attachment to macroscale area of interest.
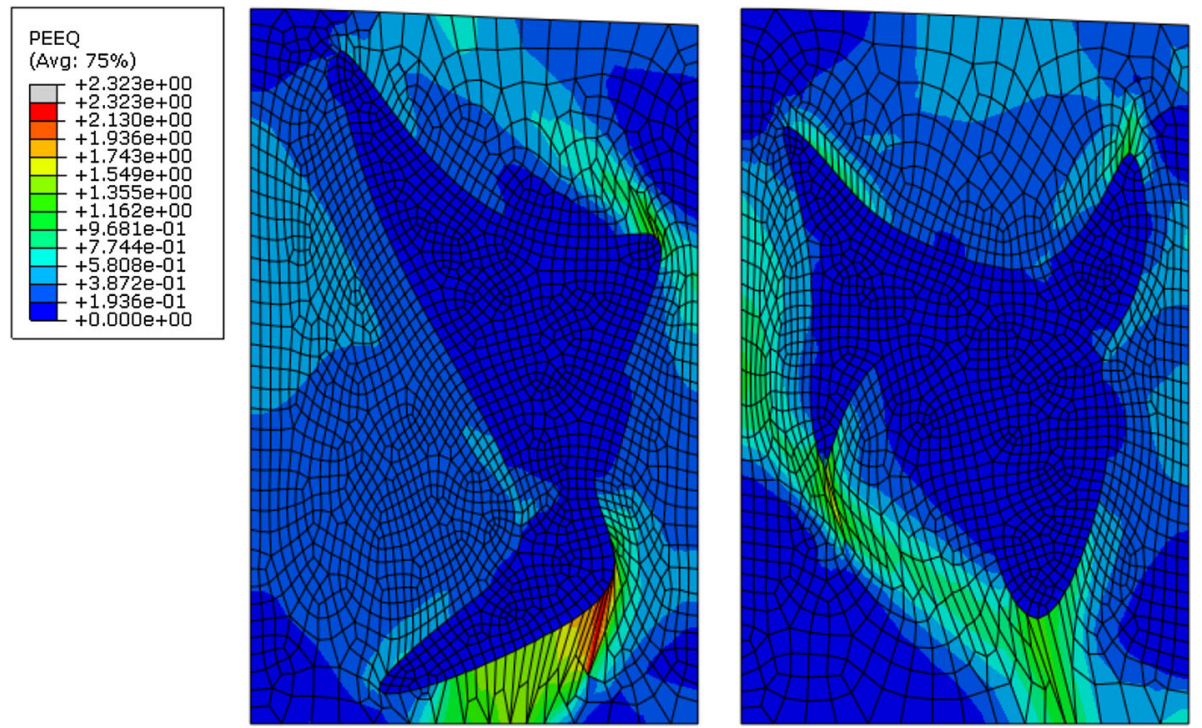

Fig. 12-Effective strain calculated for SSRVEs attached to selected macro element. 
change of the thickness of the strip calculated by the macro FE model of stamping. Finally, the FE calculations in microscale are performed. The idea of SSRVE attachment to macroscale is presented in Figure 11.

\section{DISCUSSION}

The process of initial stamping during the crash box manufacturing cycle was selected for a deeper analysis by using multiscale simulations. Two different SSRVEs (presented in Figure 10), obtained for HTC600 DP steel, were applied as a basis for microscale simulations. The results shown in Figures 12 and 13 present a distribution of effective strains and stresses. However, it can be seen that for different shapes of SSRVEs, different values of maximum strain and stress are obtained. This suggests that an additional factor in the form of strain and stress distributions should be incorporated into an objective function during SSRVE procedure. It will allow the determination of the best unambiguous solution and will lead to more reliable simulations.

The computing times were measured for all simulations in microscale and the following results were obtained dependently on the number of finite elements:

- 7-point SSRVE

- 1072 elements - 11 seconds

- 1798 elements-14 seconds

- 10-point SSRVE

- 849 elements - 10 seconds

- 2262 elements - 25 seconds

- 13-point SSRVE

- 813 elements - 8 seconds

- 1949 elements - 20 seconds

The measured time of calculations is strongly related to the number of finite elements. Therefore, numerical simulations using real microstructure were performed to com-
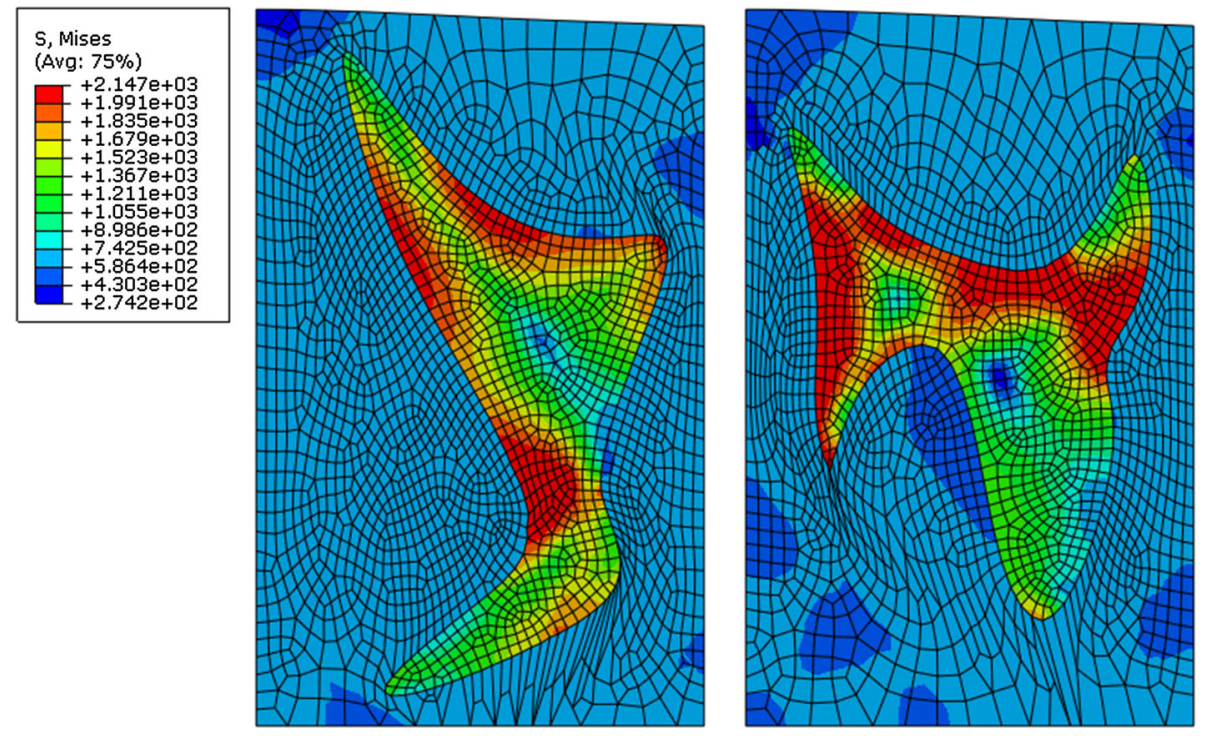

Fig. 13-Effective stress [MPa] calculated for different SSRVEs attached to selected macro element.

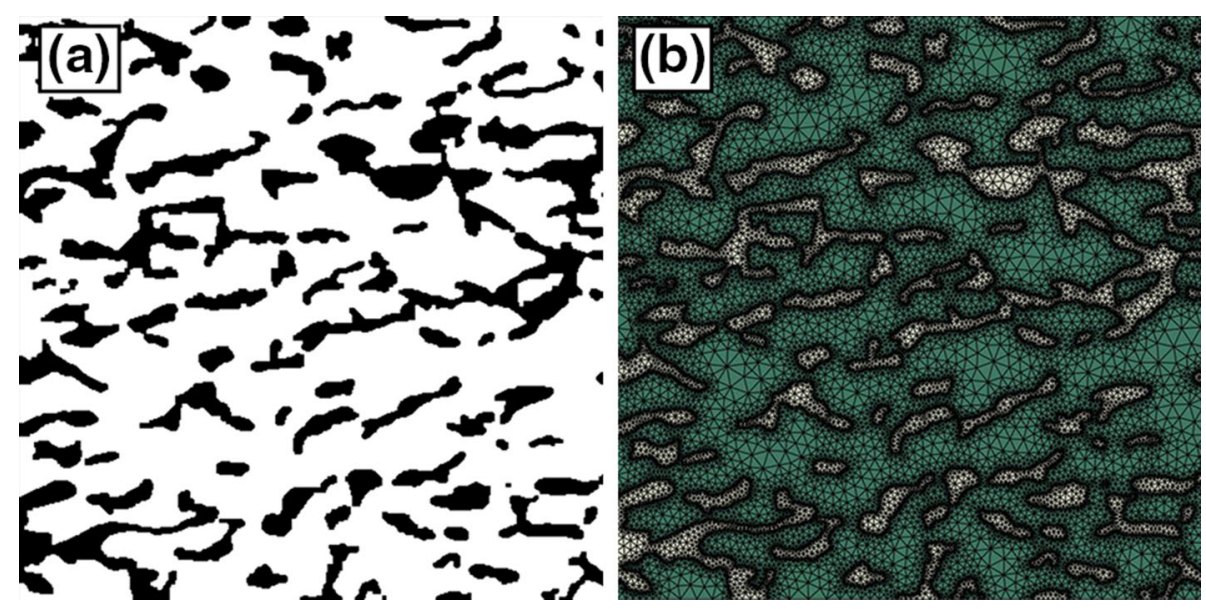

Fig. 14-Effective stress [MPa] calculated for different SSRVEs attached to selected macro element. 
pare obtained timings with its related to SSRVE. The extended version of microstructure presented in Figure 9 was used for this purpose. Its segmented and meshed versions are presented in Figures 14(a) and (b), respectively.

This computational characteristic of applied meshed microstructure are as follows:

- Original microstructure

- 96,549 elements-308 seconds

It is clearly visible that even SSRVE composed of many NURBS control points is much faster than sophisticated microstructure. Simultaneously the quality of the obtained results is maintained.

\section{CONCLUSIONS}

An analysis of the computing times and predictive capabilities of the models allowed to conclude that the modified Avrami equation gives good results as far as the prediction of volume fractions after annealing is needed. Optimization of the laminar cooling and continuous annealing for DP steels is possible using a connection of this equation with the FE solution for the macro temperature field. In contrast, a multiscale model with microstructure taken explicitly into consideration is needed to analyze distributions of strains in the ferriticmartensitic microstructure. This approach is computationally very expensive. However, it is shown in this article that application of the statistically similar representative element allows to decrease the computing times even by more than an order of magnitude, while the accuracy and predictive capabilities remain almost unchanged.

The results of simulation of the cycle composed of cold rolling, continuous annealing, and stamping to manufacture parts for the automotive industry are presented in this article.

\section{ACKNOWLEDGMENTS}

Financial assistance from the NCBiR, project no. NR07-0053-10, is acknowledged.

\section{OPEN ACCESS}

This article is distributed under the terms of the Creative Commons Attribution License which permits any use, distribution, and reproduction in any medium, provided the original author(s) and the source are credited.

\section{REFERENCES}

1. M. Avrami: J. Chem. Phys., 1939, vol. 7, p. 1103.

2. W. Johnson and R. Mehl: Trans. AIME, 1939, vol. 135, p. 416.

3. A. Kolmogorov: I.A. Nauk USSR Ser. Matemat., 1937, vol. 3, p. 355 .

4. B. Donnay, J.C. Herman, V. Leroy, U. Lotter, R. Grossterlinden, and H. Pircher: Proc. $2^{\text {nd }}$ Conf. Modelling of Metal Rolling Processes, J.H. Beynon, P. Ingham, H. Teichert, and K. Waterson, eds., London, U.K., 1996, pp. 23-35.

5. J.P. Simmons, C. Shen, and Y. Wang: Scripta Mater., 2000, vol. 43, p. 935 .

6. M. Militzer: Curr. Opin. Solid State Mater. Sci., 2011, vol. 15, p. 106 .

7. M. Pietrzyk and R. Kuziak: Proc. $2^{\text {nd }}$ ESAFORM Conf., J. Covas, ed., Guimaraes, Portugal, 1999, pp. 525-28.

8. M. Pernach and M. Pietrzyk: Comput. Mater. Sci., 2008, vol. 44, p. 783.

9. L. Madej, A. Mrozek, W. Kuś, T. Burczyński, and M. Pietrzyk: Comput. Methods Mater. Sci., 2008, vol. 8, p. 1.

10. D. Szeliga: Comput. Methods Mater. Sci., 2012, vol. 12, p. 70.

11. D. Szeliga, J. Gawąd, and M. Pietrzyk: Comput. Methods Appl. Mech. Eng., 2006, vol. 195, pp. 6778-98.

12. M. Pietrzyk and R. Kuziak: Microstructure Evolution in Metal Forming Processes, J. Lin, D. Balint, and M. Pietrzyk, eds., Woodhead Publishing, Oxford, U.K., 2012, p. 145.

13. M. Pietrzyk, L. Madej, L. Rauch, and R. Golab: Arch. Civ. Mech. Eng., 2010, vol. 10, p. 57.

14. J. Schroeder, D. Balzani, and D. Brands: Arch. Appl. Mech., 2011, vol. 81, p. 975 .

15. L. Rauch and K. Bzowski: Comput. Methods Mater. Sci., 2011, vol. 11, p. 350.

16. L. Rauch, M. Pernach, K. Bzowski, and M. Pietrzyk: Comput. Methods Mater. Sci., 2011, vol. 11, p. 531.

17. L. Rauch and L. Madej: Int. J. Multiscale Comput. Eng., 2010, vol. 8, p. 1.

18. M. Ambrozinski, K. Bzowski, L. Rauch, and M. Pietrzyk: Arch. Civ. Mech. Eng., 2012, vol. 12, p. 126. 